\title{
Sustainability of Tobacco Treatment Programs in the Cancer Center Cessation Initiative
}

\author{
The Cancer Center Cessation Initiative Sustainability Working Group*
}

\begin{abstract}
The NCl's Cancer Center Cessation Initiative (C3I) has a specific objective of helping cancer centers develop and implement sustainable programs to routinely address tobacco cessation with patients. Sustaining tobacco treatment programs requires the maintenance of (1) core program components, (2) ongoing implementation strategies, and (3) program outcomes evaluation. $\mathrm{NCl}$ funding of C3l included a commitment of resources toward sustainability. This article presents case studies to illustrate key strategies in developing sustainability capacity across 4 C3I-funded sites. Case studies are organized according to the domains of sustainability capacity defined in the Clinical Sustainability Assessment Tool (CSAT). We also describe the C3I Sustainability Working Group agenda to make scientific and practical contributions in 3 areas: (1) demonstrating the value of tobacco use treatment in cancer care, (2) identifying implementation strategies to support sustainability, and (3) providing evidence to inform policy changes that support the prioritization and financing of tobacco use treatment. By advancing this agenda, the Sustainability Working Group can play an active role in advancing and disseminating knowledge for tobacco treatment program sustainability to assist cancer care organizations in addressing tobacco use by patients with cancer within and beyond C3I.

J Natl Compr Canc Netw 2021;19(Supp/ 1):S16-20
\end{abstract} doi: $10.6004 /$ jnccn.2021.7093

\section{Background}

NCI launched the C3I to support the implementation of tobacco treatment programs at NCI-designated Cancer Centers nationwide. ${ }^{1}$ C3I has a specific objective "to help cancer centers build and implement sustainable tobacco cessation treatment programs to routinely address tobacco cessation with cancer patients." ${ }^{1}$ C3I has demonstrated substantial progress in achieving this goal. ${ }^{2}$ Funded C3I centers were required to commit to sustaining their programs beyond initial NCI funding.

To our knowledge, no prior research has focused on the systematic sustainment (ie, continued implementation) of tobacco treatment programs in cancer care settings. ${ }^{3}$ C3I presents an ideal laboratory for examining how tobacco treatment program sustainability is operationalized in the context of clinical oncology care to achieve population-level benefits. In particular, the C3I experience can advance knowledge about the value of tobacco treatment programs in cancer care settings, the implementation strategies used to promote sustainability (ie, continued clinical benefit of the intervention) and policy change required to sustain programs and their benefits over time. The initiative can serve as a model for organizations within and beyond NCI-designated Cancer Centers looking to establish and maintain tobacco treatment services.

*A complete list of the collaborators in the Cancer Center Cessation Initiative Sustainability Working Group appears at the end of this article.

\section{Science of Clinical Sustainability}

The field of implementation science has contributed significantly to our understanding of evidence-based program adoption and implementation in cancer care. ${ }^{4}$ However, less is known about how well or under what conditions programs are sustained and their health benefits are maintained, ${ }^{5,6}$ representing a critical gap in healthcare research. ${ }^{6}$

Traditionally, sustainability has been conceptualized as the final stage of implementing an intervention. However, in recent years, it has evolved to consider adaptation of implementation processes to improve intervention fit within clinical settings and broader sociostructural contexts. ${ }^{5}$ Consideration for sustainability ideally begins as the intervention is developed.

Effective practices cannot be sustained unless their clinical and organizational systems have the capacity to maintain them over time. Sustainability capacity refers to the existence of structures and processes that enable a program to effectively implement and maintain evidence-based practices. ${ }^{7}$ Although tobacco cessation and sustained abstinence have been demonstrated to benefit patients with cancer and reduce cancer treatment costs, ${ }^{8}$ and even though documented costs-per-quit in C3I have been modest,${ }^{9}$ sustainability remains a critical challenge. Consistent with recommended definitions and conceptualizations that consider complex health interventions, ${ }^{5,6,10}$ the sustainability of tobacco treatment programs can be conceptualized in 3 critical domains: maintenance of (1) core program components 
(ie, evidence-based interventions to improve cessation; eg, counseling, pharmacotherapy), (2) ongoing implementation strategies (ie, methods to enhance the adoption, implementation, and sustainment of an intervention; eg, automated referral, opt-out referral, point-of-care treatment), and (3) program outcomes evaluation (ie, consequences of implementing the intervention; eg, reach, effectiveness).

\section{Building Sustainability Capacity}

C3I centers have demonstrated substantial progress in the implementation of evidence-based tobacco treatment programs (ie, core components) by applying a range of implementation strategies, with nearly all integrating tobacco treatment screening and referral into their electronic health record (EHR) systems and workflows. C3I metrics have also demonstrated achievement of key sustainability milestones related to training staff, devising new clinical workflows, engaging information technology leadership, and implementing billing and reimbursement approaches. Most programs have dedicated staff to deliver tobacco treatment services; many also offer referrals to external sources, such as state quitlines. These efforts have resulted in improved program reach (ie, program outcomes), including among racial/ethnic minority patients. ${ }^{11}$

To assess sustainability capacity, the C3I Coordinating Center incorporated the Clinical Sustainability Assessment Tool (CSAT) into its evaluation process. ${ }^{12}$ The CSAT is an established practice appraisal tool that encompasses 7 critical domains: engaged staff and leadership, engaged stakeholders, organizational readiness, workflow integration, implementation and training processes, monitoring and evaluation, and outcomes and effectiveness (including cost-effectiveness). ${ }^{12}$ Additionally, each C3I site is required to complete a Sustainability Plan at the conclusion of their funded period. The Sustainability Plan complements the CSAT by asking sites to outline the resources they have committed and their overall plan for sustaining their program beyond C3I funding. Case studies illustrating key strategies C3I sites are using to develop sustainability capacity area presented in Table 1 . Cases represent a convenience sample of 4 centers across the 3 C3I cohorts, which entered the initiative in 2017, 2018, and 2020, respectively. These case studies illustrate implementation strategies for sustaining gains within the CSAT.

To engage staff and leadership, all 4 centers formed multidisciplinary implementation teams, which include cancer center leadership, clinical champions, and tobacco treatment care teams. The centers also engaged internal and external stakeholders, including information technology (IT) personnel, data analysts, pharmacists, patients, and families. All 4 centers have made progress in monitoring and evaluation following guidance from C3I on standardized metrics for tobacco assessment and treatment outcomes. The centers developed training programs and materials targeted to tobacco treatment program and oncology care team staff as well as oncology patients. All 4 centers leveraged EHR-based registries to automate the identification of patients using tobacco, including IT tools to disseminate patient screening and referral to tobacco treatment programs through tobacco registries. All sites have taken steps to optimize workflow integration using in-clinic observations and EHR-based solutions, and to improve cancer center organizational readiness in support of tobacco treatment programs.

\section{Sustainability Working Group Agenda}

The C3I Coordinating Center and participating clinicians, researchers, and staff formed the Sustainability Working Group (SWG). Through monthly and ad hoc meetings, the SWG helps strengthen sustainable tobacco use treatment in cancer care by developing and disseminating products to advance the science and practice of program sustainability in 3 areas: (1) demonstrating the value of tobacco use treatment in cancer care, (2) identifying implementation strategies to support sustainability, and (3) providing evidence to inform policy changes that support the prioritization and financing of tobacco use treatment.

Given the increasing societal spending on cancer care, the uncertain and disparate access to care across oncology settings, and the substantial financial burdens facing patients with cancer, demonstrating the value of tobacco treatment services in cancer care is of critical importance. Undera narrowly defined fee-for-service paradigm, offering tobacco treatment in oncology settings results in a net loss in clinical revenue despite evidence that quitting smoking improves cancer outcomes. ${ }^{13}$ Shifting this paradigm to focus more broadly on value and quality of healthcare can capture both the clinical importance of tobacco cessation interventions within patient care and the relative costs of tobacco treatment in proportion to its benefits. Research is needed to determine how much is gained when effective tobacco treatment programs are sustained, and the cost-benefit trade-offs. ${ }^{14}$ The demonstrable value of tobacco treatment programs can inform system-level change that is needed to support the sustainability of tobacco treatment services in cancer care. The SWG will advance approaches to increasing sustained institutional support for tobacco treatment programs by demonstrating economic advantages and the important impact of tobacco use treatment on organizational quality of care metrics. For example, the Commission on Cancer accreditation could help prioritize tobacco use assessment and treatment as a cancer care standard, ${ }^{15}$ and advocacy organizations can approach legislators about funding programs to address health disparities in tobacco treatment by supporting outreach programs and demonstration projects. 


\section{Table 1. Strategies Used by Select Cancer Centers to Enhance Sustainability Capacity}

Cancer Center (Years of C3I Funding)

\section{Strategies Organized}

by CSAT Domain

\section{University of Virginia
Cancer Center (2017-2019)}

\section{Engaging staff and leadership}

Convening multidisciplinary teams

Providing updates to clinic staff

and seeking feedback regularly

Convening IT systems and/or programmatic working groups

Achieving leadership buy-in

\section{Engaging stakeholders}

Developing strategic external partnerships

Convening patient and family advisory board

Conducting routine patient satisfaction surveys

Conducting provider surveys to

assess challenges

\section{Monitoring and evaluation}

Developing EHR-based program metrics reporting

Developing EHR-based

tobacco registry

Tracking implementation and

maintenance costs

\section{Implementation and training}

\section{Developing training materials and} webinars for clinicians

Developing patient audiovisual materials and/or information sheets

Developing roles and responsibilities assignment matrix

Developing outreach and assessment scripts for TTS

Sponsoring TTS training for clinical champions

\section{Outcomes and effectiveness}

Measuring program effectiveness

using patient self-report in EHR

\section{Workflow integration}

$\square$

$\square$

$\square$

$\square$ $\square$

$\square$ $\square$

$\square$

$\square$ $\square$

$\square$ $\square$

$\square$ $\square$

$\square$

$\square$ $\square$
Fox Chase Cancer Center (2020-2021)

\section{tanford University (2018-2021)}

$\square$

$\square$

$\square$

$\square$

$\square$

$\square$ $\square$
Conducting workflow observations

Developing scalable EHR workflow

\section{Organizational readiness}

Establishing support systems for program functions

Fostering partnership with complementary initiatives

Securing leadership commitment for continued funding

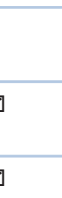

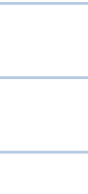


The SWG is also interested in investigating which implementation strategies are most effective for sustaining tobacco treatment programs. Investigating this empirical question will extend previous research identifying such strategies, ${ }^{16}$ with ongoing work in C3I to better understand the role of implementation strategies in optimizing clinical outcomes. The SWG recognizes the importance of synthesizing and disseminating lessons learned to cancer care organizations beyond C3I. The SWG is developing guidance for sustainability strategies within a best practice implementation guide involving collaboration across C3I working groups.

Policy-oriented research questions involve advancing the field with respect to the cost-effectiveness of tobacco treatment, determining the costs of new strategies relative to improved outcomes, and changing reimbursement policies to support the financial sustainability of tobacco treatment. The SWG will develop new evidence on the cost-effectiveness of tobacco treatment in cancer care to inform approaches for public and private payers to more adequately reimburse for tobacco treatment through bundled or fee-for-service payments. The SWG will also develop guidance for advocacy organizations and oncology societies.

\section{Conclusions}

C3I has catalyzed tobacco treatment in the cancer care setting nationwide, and now presents a unique opportunity for an ongoing learning system. Tobacco treatment programs in the C3I will continue to adapt and evolve over time. As C3I transitions beyond the initial implementation phase, there is a unique opportunity to assess how tobacco treatment program components are sustained over time, the role of implementation strategies in promoting sustainability, and how the health impacts of these programs are sustained. By advancing research into the critical domains of sustainability aligned with a policy and practice agenda to promote sustainability, the SWG will play an active role in synthesizing and disseminating knowledge gained through this system to benefit cancer care organizations within and beyond C3I.

\section{Cancer Center Cessation Initiative Sustainability Working Group Members}

Ramzi G. Salloum, PhD ${ }^{1, *}$; Linda Fleisher, PhD, MPH ${ }^{2, *}$; Sarah D. Hohl, MPH, $\mathrm{PhD}^{3,+}$; Sarah V. Clark, BA ${ }^{4,+}$; Elizabeth Klass, RN, BSN ${ }^{4, t}$; Neely A. Dahl,
$\mathrm{MPH}^{5,+}$; Brittany Pike, MS, RN ${ }^{6, t} ;$ Katie L. Lenhoff, MPH ${ }^{7, \neq} ;$ Cary A. Presant, MD FACP, FASCO ${ }^{8, \neq}$; Kimberly A. Shoenbill, MD, PhD ${ }^{9,7}$; Alex T. Ramsey, PhD ${ }^{10, \neq}$ Thulasee Jose, MD ${ }^{11, \pm} ;$ Andrew T. Day, MD, MPH ${ }^{12, \neq} ;$ Michael Dark, MPH ${ }^{13,+}$ Sophia Yeung, MHA ${ }^{8, \neq}$; Elisa K. Tong, MD ${ }^{14, \neq}$; Judith J. Prochaska, PhD ${ }^{6, \neq}$ Jamie S. Ostroff, PhD ${ }^{15, \neq}$; Donna Shelley, MD, MPH ${ }^{16, \neq} ;$ Graham W. Warren $\mathrm{MD}, \mathrm{PhD}^{17, \neq}$; Robert Adsit, MEd ${ }^{18, \$}$; Mara Minion, $\mathrm{MA}^{3, \$}$; Danielle Pauk, $\mathrm{BS}^{3, \S}$; and Betsy Rolland, PhD, MLIS, MPH ${ }^{3,19, \$}$

${ }^{1}$ Department of Health Outcomes and Biomedical Informatics, University of Florida College of Medicine, and University of Florida Health Cancer Center Gainesville, Florida; ${ }^{2}$ Fox Chase Cancer Center, Philadelphia, Pennsylvania; ${ }^{3}$ Carbone Cancer, School of Medicine and Public Health, University of Wisconsin-Madison, Madison, Wisconsin; ${ }^{4}$ Department of Preventive Medicine, Feinberg School of Medicine, and Robert H. Lurie Comprehensive Cancer Center of Northwestern University, Chicago, Illinois; ${ }^{5}$ University of Virginia Cancer Center, Charlottesville, Virginia; ${ }^{6}$ Stanford Cancer Institute, Stanford, California; ${ }^{7}$ Dartmouth-Hitchcock Norris Cotton Cancer Center, Lebanon, New Hampshire; ${ }^{8}$ Department of Medical Oncology and Therapeutics Research, City of Hope Comprehensive Cancer Center, and Beckman Research Institute, Duarte, California; ${ }^{9}$ Department of Family Medicine, School of Medicine, University of North Carolina at Chapel Hill, Chapel Hill, North Carolina; ${ }^{10}$ Department of Psychiatry, Washington University in St. Louis School of Medicine, St. Louis, Missouri; ${ }^{11}$ Department of Anesthesiology and Perioperative Medicine, Mayo Clinic, Rochester, Minnesota; ${ }^{12}$ Department of Otolaryngology - Head and Neck Surgery, University of Texas Southwestern Medical Center, and Simmons Comprehensive Cancer Center, Dallas, Texas; ${ }^{13}$ Department of Family and Community Medicine, School of Medicine, University of Maryland, Baltimore, Maryland; ${ }^{14}$ Department of Internal Medicine; University of California Davis Comprehensive Cancer Center, Sacramento, California; ${ }^{15}$ Department of Psychiatry and Behavioral Sciences, Memorial Sloan Kettering Cancer Center, New York, New York; ${ }^{16}$ Department of Public Health Policy and Management, and Global Center for Implementation Science, New York University School of Global Public Health, New York, New York; ${ }^{17}$ Department of Radiation Oncology, and Department of Cell and Molecular Pharmacology and Experimental Therapeutics, Hollings Cancer Center, Medical University of South Carolina, Charleston, South Carolina; ${ }^{18}$ Department of Medicine, Center for Tobacco Research and Intervention, School of Medicine and Public Health, University of Wisconsin-Madison, Madison, Wisconsin; and ${ }^{19}$ Institute for Clinical and Translational Research, University of Wisconsin-Madison, Madison, Wisconsin.

*Working Group Chair; 'Writing Group Author; ${ }^{*}$ Working Group Author; ${ }^{\S}$ Coordinating Center Author.

Submitted July 26, 2021; final revision received September 14, 2021; accepted for publication September 16, 2021.

Disclosures: E.K. Tong has disclosed participating in research for the California Tobacco Control Program (Principal Investigator). J.J. Prochaska has disclosed serving on a data safety monitoring board for Achieve Life Science, serving on an advisory board for Carrot, acting as plaintiffs' expert witness in lawsuits against tobacco companies, and owning stock in Carrot. G.W. Warren has disclosed participating in research for the Agency for Healthcare Research and Quality and serving as a consultant for the Canadian Partnership Against Cancer. The remaining individuals have disclosed no relevant financial relationships.

Funding: This supplement was funded by the C3I Coordinating Center contract from the National Cancer Institute (CRDF Award \#66590). In addition, authors received funding for their $\mathrm{C} 3 \mathrm{I}$ participation via a supplement to their NCI P30 cancer center support grant during the period 2017-2021.

Correspondence: Ramzi G. Salloum, PhD, University of Florida, College of Medicine, 2004 Mowry Road, Gainesville, FL 32610. Email: rsalloum@ufl.edu

\section{References}

1. Croyle RT, Morgan GD, Fiore MC. Addressing a core gap in cancer care - the $\mathrm{NCl}$ Moonshot program to help oncology patients stop smoking. N Engl J Med 2019;380:512-515.

2. D'Angelo $H$, Rolland $B$, Adsit $R$, et al. Tobacco treatment program implementation at $\mathrm{NCl}$ Cancer Centers: progress of the $\mathrm{NCl}$ Cancer Moonshot-funded Cancer Center Cessation Initiative. Cancer Prev Res (Phila) 2019;12:735-740. 
3. Berta WB, Wagg A, Cranley L, et al. Sustainment, Sustainability, and Spread Study (SSaSSy): protocol for a study of factors that contribute to the sustainment, sustainability, and spread of practice changes introduced through an evidence-based quality-improvement intervention in Canadian nursing homes. Implement Sci 2019;14:109.

4. Neta G, Clyne M, Chambers DA. Dissemination and implementation research at the National Cancer Institute: a review of funded studies (2006-2019) and opportunities to advance the field. Cancer Epidemiol Biomarkers Prev 2021;30:260-267.

5. Shelton RC, Cooper BR, Stirman SW. The sustainability of evidencebased interventions and practices in public health and health care. Annu Rev Public Health 2018;39:55-76.

6. Scheirer MA, Dearing JW. An agenda for research on the sustainability of public health programs. Am J Public Health 2011;101:2059-2067.

7. Schell SF, Luke DA, Schooley MW, et al. Public health program capacity for sustainability: a new framework. Implement Sci 2013;8:15.

8. Warren GW, Cartmell KB, Garrett-Mayer E, et al. Attributable failure of first-line cancer treatment and incremental costs associated with smoking by patients with cancer. JAMA Netw Open 2019;2:e191703.

9. Salloum RG, D'Angelo H, Theis RP, et al. Mixed-methods economic evaluation of the implementation of tobacco treatment programs in National Cancer Institute-designated cancer centers. Implement Sci Commun $2021 ; 2: 41$
10. Jolles MP, Lengnick-Hall R, Mittman BS. Correction to: core functions and forms of complex health interventions: a patient-centered medical home illustration [published online April 1, 2019]. J Gen Intern Med,

11. D'Angelo $\mathrm{H}$, Webb Hooper $\mathrm{M}$, Burris $\mathrm{JL}$, et al. Achieving equity in the reach of smoking cessation services within the $\mathrm{NCl}$ Cancer Moonshot-funded Cancer Center Cessation Initiative. Health Equity 2021;5:424-430.

12. Malone S, Prewitt K, Hackett R, et al. The Clinical Sustainability Assessment Tool: measuring organizational capacity to promote sustainability in healthcare. Implement Sci Commun 2021;2:77

13. US Department of Health and Human Services. Smoking cessation: a report of the Surgeon General. Accessed September 6, 2021. Available at: https://www.hhs.gov/sites/default/files/2020-cessation-sgr-full-report. pdf

14. Proctor E, Luke D, Calhoun A, et al. Sustainability of evidence-based healthcare: research agenda, methodological advances, and infrastructure support. Implement Sci 2015;10:88.

15. Tong EK, Wolf T, Cooke DT, et al. The emergence of a sustainable tobacco treatment program across the cancer care continuum: a systems approach for implementation at the University of California Davis Comprehensive Cancer Center. Int J Environ Res Public Health 2020;17: E3241.

16. Hailemariam M, Bustos T, Montgomery B, et al. Evidence-based intervention sustainability strategies: a systematic review. Implement Sci 2019;14:57. 